\title{
Dynamic Subcarrier Allocation for OFDMA-PONs with Monitoring Mechanism
}

\author{
Wansu Lim, Pandelis Kourtessis, Milos Milosavljevic, John M. Senior \\ Optical Networks Group, Science and Technology Research Institute (STRI), University of Hertfordshire \\ College Lane Campus, Hatfield, AL10 9AB, UK \\ \{w.lim9, p.kourtessis, m.milosavljevic, j.m.senior\}@herts.ac.uk
}

\begin{abstract}
A new protocol design for 10G OFDMA-PONs is reported, demonstrating dynamic subcarrier allocation based on monitoring of each ONU's queuing status. $0.7 \mathrm{~ms}$ packet delay and $540 \mathrm{Mbps}$ throughput were achieved for SLA 0 ONUs.
\end{abstract}

OCIS codes: (060.4510) Optical communication; (060.4250) Networks

\section{Introduction}

The increasing demands of modern applications such as HDTV, 3DTV and ultra-high speed browsing lately lead the research towards increased-capacity optical access solutions. To that extent, a few studies, with relevance to access networks, have recently been presented which prove that orthogonal frequency division multiplexing (OFDM) can provide high capacity, long reach and cost-effective operation for passive optical networks (PONs) [1-4]. The upstream OFDMA subcarrier allocation in these architectures could however result in data collisions and reduced bandwidth utilization. Therefore, this paper presents an efficient bandwidth allocation protocol for OFDMA-PONs with novel dynamic subcarrier allocation (DSA) algorithm utilizing different service level agreements (SLAs).

\section{Dynamic subcarrier allocation (DSA) Algorithm}

The DSA algorithm assumes non-status-reporting ONUs, by means of a Monitoring Mechanism, utilizing a monitoring window and grant messages, as opposed to report-grant messages to automatically allocate subcarriers to ONUs. Two scenarios are used by the OLT to select the subcarrier allocation mode at each window. In Scenario 1, if the subcarriers allocated in the previous window to an ONU are not fully utilized, the OLT predicts the already given subcarrier number is adequate or needs to be reduced. Consequently, the OLT subtracts the monitored subcarriers used (Used_SC), in a given window, from the allocated subcarriers in the previous window and releases the remaining to be utilized elsewhere in the network. Figure 1 represents Scenario 1 as applied for ONU 2. During the $N^{\text {th }}$ monitoring window ONU 2 profited by the allocation of 5 subcarriers, although only 2 subcarriers were practically utilized. As a result the OLT will end up reducing at monitoring window $(N+1)$ the subcarriers to be allocated to ONU 2 releasing three additional subcarriers to the network. In Scenario 2, the OLT monitors bandwidth transmission equivalent to full utilization of the previous window's allocation process, as envisaged by an ONU's full queue. In such scenario the OLT proceeds by assuming an increase in that ONU's allocated subcarriers reflect more accurately its bandwidth requirements. This is achieved by distributing the subcarriers deposited from Scenario 1 ONUs to Scenario 2 ONUs at strict SLA priority. This is exhibited in Figure 1 by ONUs 1 and 3. The higher the SLA assignment of an ONU the bigger the number of subcarriers they are reallocated if required.

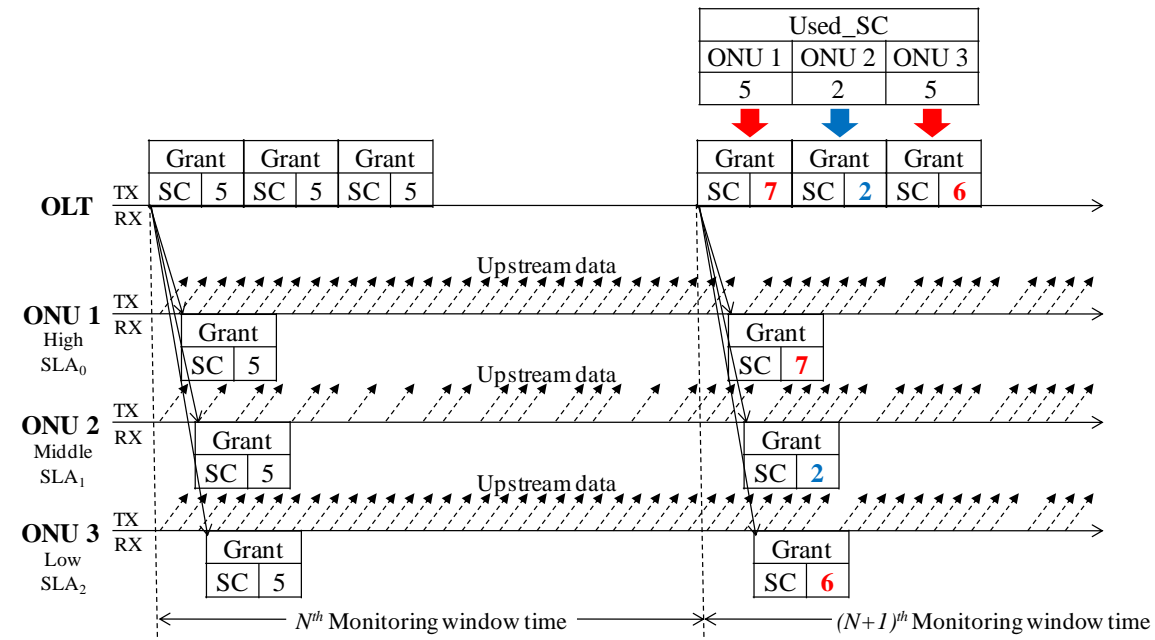

Figure 1 Example of DSA transmission with Monitoring mechanism 


\section{Simulation and results}

To evaluate the proposed algorithm in terms of the network throughput and end-to-end packet delay, an event-driven packet-based simulation model is developed using the industrial standard OPNET. The simulation model exhibits an OFDMA-PON composed of one OLT and 32 ONUs. The distance between the OLT and each ONU is $20 \mathrm{~km}$. Three SLAs, $\mathrm{SLA}_{\mathrm{t}}, \mathrm{t}=0,1,2$, from high to low priority have been considered. The number of ONUs in each service level is set to 2, 10 and 20 with the buffer size of each ONU limited to 10 MBytes. The total upstream data capacity is 10 Gbps, arranged in 64 subcarriers of $156.25 \mathrm{Mbps}$ each. In addition, the guaranteed bandwidth of the high SLA to low SLA is set to $468.75 \mathrm{Mbps}$, 312.5 Mbps and 156.25 Mbps respectively. Grant processing and propagation delays are considered at $0.5 \mu \mathrm{s}$ and $0.5 \mu \mathrm{s} / \mathrm{km}$ respectively. The network traffic is implemented by a Pareto selfsimilar traffic model with a typical Hurst parameter of 0.8 to simulate practical network patterns. The packet size is uniformly generated between 64-1518 Bytes.

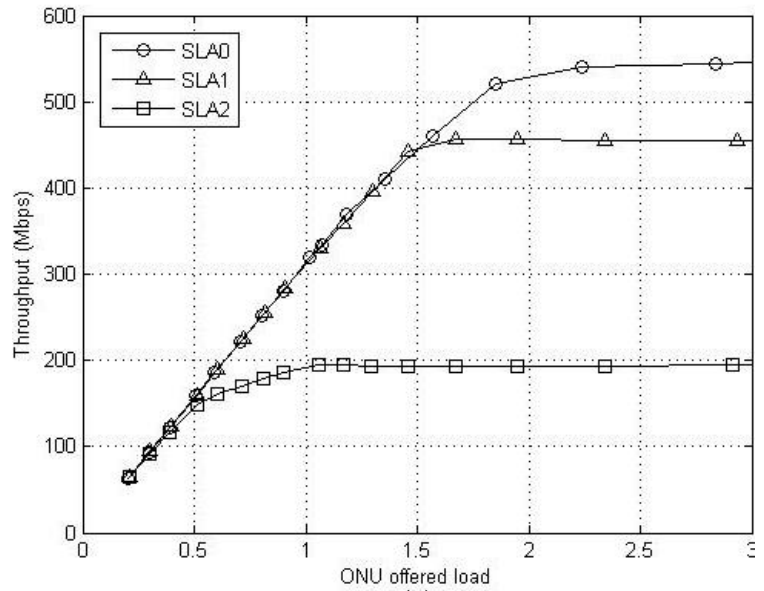

(a)

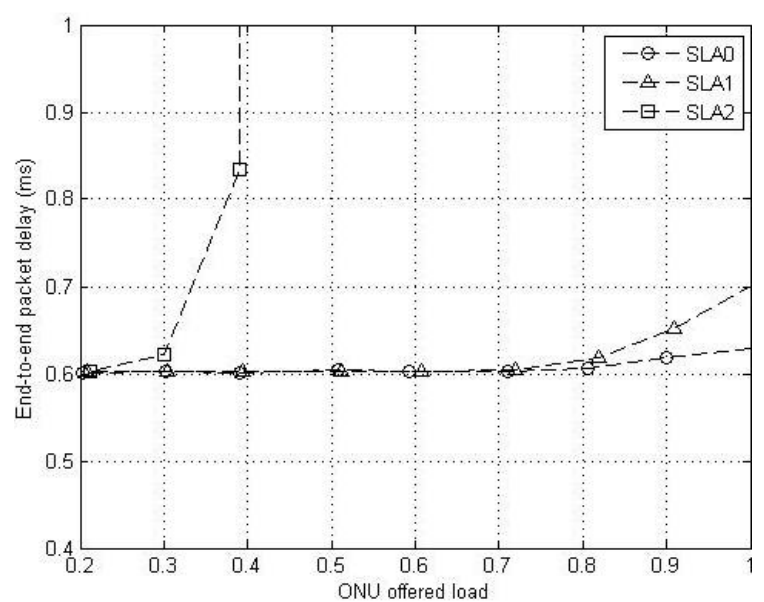

(b)

Figure 2 Simulation results according to three SLA grades (a) the network throughput, (b) the end-to-end delay.

Figure 2 (a) represents the network throughput according for all three SLAs as a function of ONU offered load and not the network load. A load of 1.0 corresponds to a data rate of $312.5 \mathrm{Mbps}$ (10 Gbps divided by $32 \mathrm{ONUs}$ ). The saturated throughput is $540 \mathrm{Mbps}, 450 \mathrm{Mbps}$ and $200 \mathrm{Mbps}$ corresponding to high to low SLAs respectively. Figure 2 (b) confirms the end-to-end packet delay of high and middle priority SLAs is less than $0.7 \mathrm{~ms}$ even if the ONU offered load is 1.0. This is because the guaranteed bandwidths of the high and middle SLAs are greater than or equal to the ONU offered load of 1.0 corresponding to $312.5 \mathrm{Mbps}$. In addition, the DSA algorithm allocates the remaining subcarriers according to the SLA priority. As expected, the end-to-end pack delay of low priority SLAs dramatically increases at an ONU offered load of 0.4 since a DSA algorithm cannot support granularity and subcarrier sharing among low bandwidth ONUs.

\section{Conclusions}

By reason of supporting increased bandwidth requirement, this paper has evaluated the performance of a new subcarrier assignment protocol and corresponding algorithm in the distinction of three SLA grades for a $20 \mathrm{~km}$ reach, 32-split OFDMA-PON. In particular, the OLT automatically monitors each ONU's queue status instead of using traditional report messaging exhibiting simplicity in MAC processing and reduced packet delays, while maintain satisfactory network throughput. The obtained end-to-end packet delay of the high and middle priority SLA is less than $0.7 \mathrm{~ms}$ at the heavy traffic loading of 1.0, but the low priority SLA is saturated at the ONU offered load of 0.4 because of the low granularity. 540 Mbps throughput were achieved for SLA 0 ONUs for a 10G OFDMAPON at 32 split.

\section{Acknowledgments}

This work was supported by the ACCORDANCE project, through the 7th ICT Framework Programme.

\section{References}

[1] Neda Cvijetic, "Advanced Digital Signal Processing (DSP) for Next-Generation OFDMA-PON,” ANIC, (Karlsruhe, Germany, 2010).

[2] M. Milosavljevic, P. Kourtessis, J. M. Senior, "Transparent wireless transmission over the ACCORDANCE optical/wireless segment," CSNDSP, (Newcastle, UK, 2010), pp. 138-142.

[3] K. Kanonakis, I. Tomkos, T. Pfeiffer, J. Prat, P. Kourtessis, "ACCORDANCE: A novel OFDMA-PON paradigm for ultra-high capacity converged wireline-wireless access networks," ICTON, (Munich, Germany, 2010).

[4] W. Wei, J. Hu, D. Qian, P. N. Ji, T. Wang, X. Liu, C. Qiao, "PONIARD: A Programmable Optical Networking Infrastructure for Advanced Research and Development of Future Internet," IEEE/OSA JLT 27, 233-241(2009). 